\title{
Treinar Memória de Trabalho Promove Mudanças em Inteligência Fluida?
}

\author{
Marcela Mansur-Alves ${ }^{1}$ \\ Laboratório de Estudos do Comportamento, Cognição e Aprendizagem (LECCA), \\ Departamento de Psicologia, Universidade Federal de Minas Gerais, \\ Belo Horizonte, MG, Brasil \\ Renata Saldanha-Silva \\ Faculdade Ciências Médicas de Minas Gerais, Belo Horizonte, MG, Brasil
}

\begin{abstract}
Resumo
A inteligência é um dos preditores mais robustos de uma variedade de resultados nos âmbitos social, acadêmico e econômico, por isso pesquisadores cada vez mais dedicam esforços para promover melhorias na inteligência. Nos últimos anos emergiu interesse em programas de treinamento em memória de trabalho como forma de promover mudanças na inteligência. Tais programas estão cercados de controvérsias. A presente pesquisa objetiva realizar uma revisão crítica de literatura, analisando os efeitos de transferência do treinamento em memória de trabalho para a inteligência fluida. Após levantamento e análise detalhada da literatura, foram reanalisados os efeitos de treino em memória de trabalho para ganhos em inteligência de 45 estudos. Como resultado, observou-se grande variabilidade metodológica entre os estudos, cuja maior parte usou amostras não clínicas, com algum incentivo material e $n<30$. $\mathrm{O}$ Raven predominou como medida de eficácia dos treinamentos em inteligência fluida, e os aumentos na mesma foram significativos para os grupos etários de crianças e treinamentos mais longos. Os efeitos positivos encontrados são insuficientes para indicar uma transferência real para inteligência fluida, o que sugere a necessidade de maior cautela no uso atual de intervenções semelhantes.
\end{abstract}

Palavras-chave: Inteligência, treino cognitivo, memória de trabalho, transferência.

\section{Does Working Memory Training Promote Changes in Fluid Intelligence?}

\begin{abstract}
Intelligence is a main predictor for a variety of social, academic, and economical outcomes. Therefore, researchers have been increasingly dedicated to pursuing ways to improve intelligence. In the recent years, a great interest in working memory training programs has risen as a form to achieve changes in intelligence. However, the efficacy of these programs is still surrounded by controversies. The current review aims to perform a critical analysis of the literature, reassessing the effects of working memory training that leads to improvements in fluid intelligence. After data collection and a detailed literature

1 Endereço para correspondência: Universidade Federal de Minas Gerais, Departamento de Psicologia, Faculdade de Filosofia e Ciências Humanas, Avenida Antônio Carlos, 6627, Campus Pampulha, Belo Horizonte, MG, Brasil 31270-901. E-mail: marcelamansuralves@yahoo.com.br ou marmansura@gmail.com Agradecimento: As autoras do trabalho agradecem ao aluno Vinícius Fernandes Diniz, do curso de graduação em psicologia da UFMG, pela colaboração na revisão do levantamento de literatura realizado para o presente artigo.
\end{abstract}


review, the effects of working memory training leading to intelligence gain from 45 studies were assessed. A great variety in methodology was observed within the studies, which used mainly non-clinical samples, with some material incentive and an $n<30$. Raven was the predominant measure of efficacy for training in fluid intelligence, and the improvements in intelligence were significant for groups of children of similar age range and for longer training periods. The positive effects observed are still insufficient to indicate a transfer to fluid intelligence, suggesting that a more cautious approach of the current similar interventions may be necessary.

Keywords: Intelligence, cognitive training, working memory, far transfer.

\section{¿Entrenar Memoria de Trabajo Promueve Cambios en la Inteligencia Fluida?}

\section{Resumen}

La inteligencia predice resultados diversos en la vida social, académico y económico, por lo que los investigadores cada vez más esfuerzos dedican a promover cambios positivos en la inteligencia. En los últimos años surgió el interés por los programas de entrenamiento de memoria de trabajo con el fin de promover cambios en la inteligencia. Dichos programas están rodeados de controversia. Esta investigación tiene como objetivo realizar una revisión crítica de la literatura sobre los efectos del entrenamiento en la memoria de trabajo para la inteligencia fluida. Después de la inspección y el análisis detallado de la literatura, fueron reanalizados los efectos de 45 estudios. Como resultado, existe una gran variabilidad metodológica entre los estudios, la mayoría de los cuales utilizan muestras no clínicas, con un poco de estímulo material y $n<30$. El Raven era la medida predominante de la eficacia. Los aumentos fueron significativos para los grupos de los niños y entrenamientos de mayor duración. Los efectos positivos encontrados son insuficientes para indicar una transferencia real para la inteligencia fluida, lo que sugiere la necesidad de una mayor precaución en el uso actual de intervenciones similares.

Palabras clave: Inteligencia, entrenamiento cognitivo, memoria de trabajo, transferencia.

A literatura científica em treinamento cognitivo vem recebendo uma explosão de interesse recente, majoritariamente decorrente das constantes afirmativas de plasticidade da memória de trabalho (MT; Astle, Barnes, Baker, Colclough, \& Woolrich, 2015). A memória de trabalho - capacidade de armazenar e manipular a informação por curtos períodos de tempo - é um importante preditor de desempenho escolar, estando também envolvida com o raciocínio, a solução de problemas matemáticos, a manutenção do foco de atenção, a tomada de decisões, a leitura e o pensamento abstrato (Baddeley, 2003; Ilkowska \& Engle, 2010). O interesse da comunidade científica é ainda maior quando se consideram resultados de estudos apontando que os ganhos observados em programas de treinamento de MT podem ser transferidos para habilidades ou processos cognitivos não dire- tamente treinados nestes programas (fenômeno conhecido como far transfer), como, por exemplo, atenção, funções executivas ou inteligência fluida (Gf; Buschkuehl \& Jaeggi, 2010; Jaeggi, Buschkuehl, Jonides, \& Perrig, 2008; Klingberg, 2010; Klingberg et al., 2005; Klingberg, Forssberg, \& Westerberg, 2002). De especial interesse para os propósitos do presente artigo, estão os possíveis ganhos observados em testes de inteligência fluida. Do ponto de vista psicométrico, a inteligência fluida é a capacidade de fazer analogias, raciocinar e resolver problemas novos, extrair significados e se adaptar a situações não usuais (Jensen, 1998). É usualmente medida através de testes de raciocínio figurativo, indutivo e analógico, classificação, e de testes de matrizes, tais como o teste das Matrizes Progressivas de Raven, que é um dos instrumentos mais utilizados para se operacionalizar $\mathrm{G} f$. Um núme- 
ro significativo de estudos aponta que, dentre os fatores intelectuais mais amplos, $\mathrm{G} f$ é o que mais se aproxima da concepção teórica do fator $g$, como proposto por Spearman (Jensen, 1998), inclusive, alguns pesquisadores acreditam que inteligência fluida e inteligência geral sejam construtos praticamente equivalentes (Ackerman, Beier, \& Boyle, 2005), pressuposição esta que tem sido parcialmente questionada por trabalhos recentes (Gignac, 2015). Ademais, G $f$ prediz de maneira consistente e robusta distintos aspectos do funcionamento individual, como desempenho e permanência escolar, desempenho ocupacional, renda mensal, comportamento disruptivo, doenças neurodegenerativas, saúde física, dentre outras (Neisser, Fuchs, \& Moreira, 1996; Nisbet et al., 2012). Um padrão consistente de semelhanças psicométricas, clínicas e neuroanatômicas fornece a base para a formulação da premissa de que o treinamento em MT poderia, assim, aumentar o desempenho em testes de inteligência fluida. É extenso o número de estudos que apontam que há associação moderada a elevada (acima de 0,50) entre diferentes tarefas de MT e medidas de inteligência fluida, indicando que ambos poderiam compartilhar uma estrutura subjacente comum (Kyllonen \& Christal, 1990; Oberauer, Suß, Schulze, Wilhelm, \&Wittmann, 2000; Orzechowski, 2010; Stauffer, Ree, \& Caretta, 1996). Existem também semelhanças do ponto de vista neuroanatômico e clínico. Estudos de neuroimagem, com amostras clínicas e não-clínicas, têm indicado que tanto as tarefas de memória de trabalho quanto os testes de inteligência fluida recrutam circuitos localizados nos lobos frontais e parietais, sendo o recrutamento moderado pela dificuldade e familiaridade com a tarefa (Baddeley, 2003; Colom, Jung, \& Haier, 2007; Jung \& Haier, 2007; Kane \& Engle, 2002; Miller \& Cohen, 2001). Se estes estudos estiverem corretos, as implicações sociais, educacionais e profissionais são consideráveis, haja vista extensa literatura apontando que a inteligência é o melhor preditor individual de diversos fenômenos sociais (Strenze, 2007). Exatamente por isso, os programas de treinamento cognitivo movimentam hoje uma indústria bilionária (Hayes, Petrov, \& Sederberg, 2015).
O debate acerca da maleabilidade da inteligência é antigo, remontando ao século XIX e aos trabalhos de Francis Galton, que apontavam para elevada influência da genética nas diferenças individuais em inteligência (Galton, 1883). Pensadores mais modernos também são céticos quanto à possibilidade de mudanças na inteligência, ressaltando sua elevada herdabilidade e estabilidade ao longo do ciclo vital. Esta visão, predominante há 30 anos, encontra embasamento nos resultados de programas de intervenção clássicos, para os quais o efeito é nulo ou de pequena magnitude, desaparecendo, muitas vezes, quase que completamente, com o transcorrer do tempo (Hernstein \& Murray, 1994; Jensen, 1981).

Entretanto, evidências recentes têm sugerido que a inteligência pode ser maleável. Um sólido corpo de conhecimento acumulado sobre os ganhos geracionais em inteligência - fenômeno conhecido como Efeito Flynn - parecem apontar que fatores ambientais diversos (ex.: nutrição, industrialização, escolarização, avanços tecnológicos, dentre outros) poderiam ter efeitos benéficos na inteligência, mesmo que a longo prazo (Flynn, 1987, 2006). Nesse contexto, emergiram também resultados de programas de intervenção mostrando que a memória de trabalho poderia ser o veículo para a modificação da inteligência (Alloway, Bibile, \& Lau, 2013; Borella, Carretti, Zanoni, Zavagnin, \& De Beni, 2013; Borella et al., 2014; Jaeggi et al., 2008; Jaeggi, Buschkuehl, Jonides, \& Shah, 2011; Jaušovec \& Jaušovec, 2012; Klingberg et al., 2005; Klingberg et al., 2002; Rudebeck, Bor, Ormond, O'Reilly, \& Lee, 2012; Schmiedek, Lövdén, \& Lindenberger, 2010; Stephenson \& Halpern, 2013; Zinke et al., 2014). O pressuposto básico destes programas é colocar o sistema de MT dos participantes funcionando constantemente e ao nível máximo a fim de estimular um aumento em seu funcionamento, o qual poderia ser transferido para outras tarefas e sistemas cognitivos, desde que compartilhassem com a MT alguma semelhança ou para cujo funcionamento a integridade da MT fosse fundamental (Au et al., 2015; von Bastian \& Eschen, 2016). Esta possibilidade, à primeira vista tão atraente para os pesquisadores, fez com que 
fossem desenvolvidos programas de intervenção em MT para diferentes faixas etárias, com desenvolvimento típico ou não.

Embora tenha havido sucesso inicial, os resultados de mais de dez anos de pesquisas permanecem, ainda, contraditórios no que diz respeito à transferência dos ganhos do treinamento em MT para inteligência (Melby-Lervåg \& Hulme, 2013; Redick, Shipstead, Wiemers, Melby-Lervåg, \& Hulme, 2015; Shipstead, Redick, \& Engle, 2012). Alguns autores relatam resultados favoráveis, apontando aumento da inteligência fluida (Borella, Carretti, Riboldi, \& De Beni, 2010; Borella et al., 2014; Jaeggi et al., 2008; Jaeggi et al., 2011; Rudebeck et al., 2012; Zinke et al., 2014) enquanto outros não encontram tais evidências e permanecem cautelosos quanto a até que ponto a plasticidade intelectual pode ser mantida após término do treinamento e se pode ser gerada por intervenções tão breves e pontuais (Chooi \& Thompson, 2012; Harrison et al., 2013; Melby-Lervåg \& Hulme, 2013, 2015; Pugin et al., 2015; Shipstead et al., 2012; Thompson et al., 2013). A comparação entre os resultados encontrados por diferentes grupos de pesquisa complica ainda mais o panorama da área, uma vez que a metodologia empregada em tais estudos é extremamente diversificada, variando desde a tarefa de MT utilizada para treinamento ao tempo total e duração do mesmo (Au et al., 2015; Buschkuehl \& Jaeggi, 2010; Melby-Lervåg \& Hulme, 2013, 2015; Redick et al., 2015). Sem uma análise detalhada dos estudos publicados sobre o assunto, qualquer conclusão sobre os benefícios dos programas de treinamento em MT para a inteligência é prematura.

Revisões sistemáticas têm sido importantes para fornecer uma análise mais pormenorizada dos estudos publicados e permitir uma clarificação dos efeitos dos programas de treinamento. A sistematização dos estudos possibilita uma reanálise dos dados, controlando os fatores que variam entre os mesmos. As revisões sistemáticas publicadas sobre o assunto buscam, de maneira geral, analisar efeitos de treinamento em tarefas específicas de MT (n-back, por exemplo) ou investigam os efeitos dos programas de trei- namento para medidas de inteligência mistas ou que operacionalizam outros fatores de segunda ordem - não especificamente a inteligência fluida (ver detalhes em Au et al., 2015; Buschkuehl \& Jaeggi, 2010; Melby-Lervåg \& Hulme, 2013; Shipstead et al., 2012). Além disso, os referidos estudos não incluem artigos escritos em português, o que acaba por excluir os estudos realizados com amostras brasileiras.

Nesse sentido, objetivou-se verificar a extensão dos efeitos de treinamento em memória de trabalho para medidas de inteligência fluida, traço latente não diretamente treinado pelas tarefas utilizadas nas intervenções (far-transfer effects ou transferência de longo alcance). $\mathrm{O}$ pressuposto para que a transferência de longo alcance ocorra é a existência de processos e mecanismos subjacentes compartilhados por duas ou mais capacidades ou habilidades cognitivas. Esse é o tipo desejável de transferência e aquele que produz mudanças cognitivas significativas (Barnet \& Ceci, 2002). Ademais, como há grande variação metodológica entre os estudos publicados, objetivou-se verificar como estas diferenças entre os estudos afetam os resultados.

\section{Método}

Esta revisão foi realizada seguindo as orientações gerais da declaração PRISMA (Preferred Reporting Items for Systematic Reviews and Meta-Analyses) $)^{2}$, a qual contempla diretrizes para realização e relato de revisões sistemáticas e meta-análises. Não obstante, alguns critérios de inclusão utilizados na presente revisão foram baseados nas orientações de Melby-Lervåg e Hulme (2013) para estudos de intervenção em memória de trabalho.

O levantamento dos artigos foi realizado no período de agosto a dezembro de 2015, e os estudos selecionados deveriam utilizar algum tipo de intervenção em MT, e incluir testes padronizados de inteligência fluida (Gf) como medidas de avaliação da eficácia da intervenção. Tais testes são compostos, em sua maioria,

www.prisma-statement.org 
por estímulos não verbais, pouco dependentes de conhecimentos previamente adquiridos e da influência de aspectos culturais. As operações mentais exigidas por tarefas de inteligência fluida incluem, de acordo com Jensen (1998): a formação e o reconhecimento de conceitos, a identificação de relações complexas, o raciocínio indutivo, a compreensão de implicações e a realização de inferências. Uma vez que a análise dos estudos apontou para a existência de uma grande diversidade de medidas de $G f$ utilizadas para avaliação da eficácia da intervenção, foram incluídas na análise as médias e desvios-padrões apenas das medidas citadas em mais de $10 \%$ da amostra total de estudos incluídos. Esse critério objetivou garantir que a medida de inteligência utilizada para avaliação da eficácia fosse reconhecidamente adequada (através da quantidade de citações) para mensuração do construto.

Na presente análise, as medidas de $G f$ analisadas foram: Matrizes Progressivas de Raven (Raven), Bochumer Matrizen-Test (BOMAT), Culture Fair Intelligence Test (CFIT). Os três instrumentos são medidas de inteligência não verbal, de múltipla escolha e independentes de cultura. As Matrizes Progressivas de Raven avaliam raciocínio indutivo, através do uso de figuras abstratas, sendo que alguns dos seus itens demandam uso de habilidades viso-espaciais, com níveis crescentes de dificuldade. Em cada item, o participante é solicitado a selecionar, dentre 6 a 8 alternativas, o segmento que falta para completar um padrão maior, apresentado no topo da página. $\mathrm{O}$ instrumento possui três versões - escala colorida, escala geral e escala avançada - que variam em número de itens e complexidade de acordo com a faixa etária e nível intelectual a que se destina (Klingberg et al., 2002; Wang, Zhou, \& Shah, 2014). De maneira semelhante, o BOMAT é um teste de matrizes composto por figuras abstratas organizadas de acordo com um padrão lógico, com um de seus elementos deixado em branco, que demandam raciocínio indutivo. Os níveis de dificuldade também são crescentes. A diferença consiste em que, enquanto no Raven as matrizes são de $3 \times 3$ elementos, no BOMAT as mesmas são de $5 \times 3$, e este último é conside- rado mais difícil do que o primeiro (von Bastian, Langer, Jäncke, \& Oberauer, 2013). Já o CFIT é composto por quatro diferentes subtestes não-verbais, que incluem matrizes, classificações, condições e séries, os quais envolvem diferentes operações cognitivas, tais como reconhecimento e formação de conceitos, raciocínio indutivo e resolução de problemas. É composto por três versões, indicadas para diferentes faixas etárias e níveis intelectuais (Borella, Carretti, Zanoni, Zavagnin, \& De Beni, 2013). As análises foram realizadas separadamente para cada uma das três medidas de Gf. Uma descrição detalhada do método de busca na literatura e critérios de inclusão podem ser encontrados na Figura 1.

Para ser incluído na revisão, o estudo deveria ter delineamento experimental, com comparação entre grupo controle e experimental, alocação aleatória dos participantes em cada grupo, e uso de pelo menos um instrumento de avaliação de $G f$ no pré-teste e no pós-teste. Quando um estudo analisado incluía mais de um grupo controle (por exemplo, um grupo controle ativo e outro passivo) optou-se por analisar as médias e desvios-padrão apenas do grupo controle ativo. Isto porque sabe-se que resultados de estudos envolvendo grupo controle ativo são mais confiáveis já que, em casos de estudos de intervenção, reduzem a probabilidade de que as melhoras observadas no grupo experimental se devam a efeito placebo (Redick et al., 2015).

Os estudos que não possuíam informações de média e desvio-padrão para as medidas de $G f$ no pré-teste e no pós-teste foram excluídos das análises. $\mathrm{O}$ tamanho do efeito foi calculado utilizando o $g$ de Hedges, que representa o $d$ de Cohen corrigido para amostras pequenas (Hedges \& Olkin, 1985). O cálculo do tamanho do efeito foi realizado para estimar a diferença entre os ganhos do grupo experimental, que recebeu treinamento, e o grupo controle. Tamanhos de efeito positivos indicam ganhos maiores para o grupo experimental. Para ponderação dos resultados de acordo com a qualidade dos estudos foram calculados os pesos, que consistem no inverso da 
variância dos estudos. Os índices $g$ foram, então, multiplicados pelos pesos dos estudos, a fim de garantir que aqueles de maior qualidade - ou seja, com menor variância e maior tamanho de amostra - tenham maior representação nas análises (Neyeloff, Fuchs, \& Moreira, 2012).

Para análise do nível de heterogeneidade entre os estudos foram calculados o $\mathrm{Q}$ de Cochrane e $\mathrm{I}^{2}$. Ambos testam a hipótese nula de que todos os estudos incluídos na revisão estão medindo um mesmo tamanho de efeito (e que as variações, neste caso, se devem a erros de amostragem). Decidiu-se pelo uso das duas medidas para garantir maior rigor nas análises, haja vista que o Q de Cochrane, embora seja a medida mais usada, pode ser pouco preciso para análises com pequeno número de estudos, casos nos quais sugere-se o uso do $\mathrm{I}^{2}$ (Higgins, Thompson, Deeks, \& Altman, 2003). Para interpretação do Q de Cochrane resultados significativos indicam alta heterogeneidade. Para análise do $\mathrm{I}^{2}$ valores acima de 25 e acima de 75 são indicativos de moderada e alta heterogeneidade, respectivamente (Higgins et al., 2003). Os resultados dos testes de heterogeneidade foram usados para definição do tipo de análise de síntese dos efeitos utilizada: efeito fixo ou efeito aleatório. O primeiro tipo pressupõe a existência de homogeneidade entre os estudos e o segundo pressupõe a existência de variações entre os grupos utilizados, além dos erros de amostragem (Neyeloff et al., 2012).

Para uma análise gráfica dos resultados em Gf foram criados Gráficos de Floresta (Forest Plot), nos quais foi apresentado o tamanho de efeito em cada estudo para comparação, além do tamanho de efeito médio total (Li \& Shotton, 2013). O viés de publicação foi analisado a partir de um gráfico de funil apenas para o Raven, haja vista que para amostras com menos de 10 estudos o mesmo não é indicado (Pereira \& Galvão, 2014). Gráficos de funil (Funnel Plots) são gráficos de dispersão, com o tamanho do efeito no eixo das abscissas e precisão (inverso da variância) no eixo das ordenadas. Estudos pouco precisos estariam distribuídos simetricamente na parte larga do funil, enquanto os mais precisos estariam na parte mais estreita. Configurações diferentes do esperado (por exemplo, com maior número de estudos com resultados positivos) indicariam um viés de publicação, ou seja, que determinada parcela dos estudos - frequentemente, aqueles com resultados negativos ou não significativos - não foi encontrada por não terem sido publicados, seja por uma decisão do autor ou do setor editorial das revistas de não publicar estudos com resultados negativos ou não significativos (Pereira \& Galvão, 2014).

As seguintes variáveis moderadoras foram utilizadas para investigar a variabilidade nos resultados dos estudos:

Idade. Dividiu-se os participantes em grupos etários da seguinte maneira: crianças/adolescentes (18 anos ou menos), adultos (19 a 59 anos) e idosos (acima de 60 anos);

Intensidade do treinamento. Foram considerados de baixa intensidade os estudos com duração total menor que 8 horas e de alta intensidade os programas com duração igual ou superior a 8 horas. A classificação seguiu o critério de Melby-Lervåg e Hulme (2013).

Tipo de grupo controle. Os estudos foram divididos dois grupos, de acordo com o tipo de intervenção realizada com o grupo controle, a saber:

1. Grupo controle passivo - não foi realizada nenhuma tarefa; e

2. Grupo controle ativo - a tarefa executada pelo grupo controle foi de natureza diferente da tarefa executada pelo grupo experimental.

Tipo de tarefa utilizada para treinamento. As tarefas de MT utilizadas foram codificadas quanto ao seu conteúdo em: viso-espaciais (que não utilizaram símbolos alfa-numéricos, carregando essencialmente na alça viso-espacial), verbais (conteúdo principal carregando na alça fonológica) e ambas (quando o programa de intervenção visava trabalhar os dois sistemas simultaneamente).

Incentivo material. Os estudos foram codificados em dois grupos: aqueles cujos participantes receberam incentivos para adesão (por exemplo, dinheiro ou presentes) e aqueles cuja participação não implicava recebimento de qualquer tipo de gratificação. 
A diferença entre os tipos de prática (distribuída $\mathrm{X}$ concentrada) não pôde ser computada, haja vista que menos de três estudos por medida de efeito utilizaram prática distribuída (menos que 3 sessões por semana). O mesmo ocorreu para a comparação entre estudos realizados com amostras clínicas e não clínicas, já que menos de três estudos foram realizados com amostra clínica. Para identificação das diferenças entre os efeitos médios gerais em cada variável moderadora foram realizados testes $Z$, de comparação entre subgrupos (Borenstein, Hedges, Higgins, \& Rothstein, 2009).

\section{Resultados}

Informações detalhadas sobre todos os estudos utilizados na revisão são apresentados na Tabela 1A (Apêndice A). A maior parte dos estudos foi realizada com amostras pequenas $(n<30)$, não clínicas, e com uso de incentivo material para os participantes. Grande parte dos estudos (74\%) utilizou-se de alguma versão das Matrizes Progressivas de Raven (Colorida, Geral ou Avançada), o que denota certa concordância na escolha da medida para avaliação de eficácia dos treinamentos em MT. Os testes de heterogeneidade revelaram moderada a alta variabilidade entre os estudos, mesmo após a exclusão dos valores extremos (mais de 2 desvios-padrão afastados da média amostral). Sendo assim, as análises de tamanho médio geral de efeito foram realizadas para efeitos aleatórios (Neyeloff et al., 2012). Os resultados para análise de viés de publicação apontaram não haver viés para os estudos com uso do Raven. Tal resultado pode ser observado na Figura 1B do Apêndice B.

A Figura 2A mostra o Gráfico de Floresta com os 33 resultados encontrados no Raven $(n$ do grupo de treinamento $=800$; média $=24,24$; $D P=8,62 ; \mathrm{n}$ do grupo controle $=827$; média $=25,06 ; D P=10,57)$. O tamanho médio geral do efeito foi $0,09(0,02-0,16$, para Intervalo de Confiança de $95 \%$, com $p<0,001)$. A heterogeneidade do grupo foi elevada, com $\mathrm{Q}=121,11$ $(p<0,001)$ e $\mathrm{I}^{2}=73,58 \%$, mesmo após a exclusão do caso com valores extremos.
O Gráfico de Floresta com os resultados no CFIT é apresentado na Figura 2B ( $n$ do grupo de treinamento=194; média $=21,56 ; D P=5,79 ; \mathrm{n}$ do grupo controle $=221 ;$ média $=24,56 ; D P=8,59)$. $O$ tamanho médio geral do efeito foi $0,24(0,14-$ 0,34, para Intervalo de Confiança de 95\%, com $p<0,05)$. A heterogeneidade do grupo foi moderada, com $\mathrm{Q}=15,81(p<0,05)$ e $\mathrm{I}^{2}=49,39 \%$, mesmo após a exclusão dos casos com valores extremos.

Os efeitos no BOMAT também são apresentados na Figura $2 \mathrm{C}$ ( $n$ do grupo de treinamento $=186 ;$ média $=23,25 ; D P=6,63 ; \mathrm{n}$ do grupo controle $=216$; média $=27,00 ; D P=13,42$ ). O tamanho médio geral de efeito foi significativo e de pequena magnitude $(g=0,24 ; p<0,05)$. $\mathrm{O}$ grau de heterogeneidade entre os estudos foi médio $\left(\mathrm{Q}=10,27 ; p>0,05 / \mathrm{I}^{2}=31,87\right)$. De maneira geral, as magnitudes de efeito da diferença entre o pós-teste e o pré-teste, entre GE e GC, encontrados no Raven foram menores do que aquelas encontradas a partir do CFIT e do BOMAT. Como o número de estudos utilizando Raven é maior, não foram encontrados vieses de publicação para ele, e o mesmo é citado na literatura como uma das medidas mais consistentes, confiáveis e com maior peso fatorial em $\mathrm{G} f$ (para detalhes ver Ackerman et al., 2005; Au et al., 2015; Carpenter, Just, \& Shell, 1990; Gray \& Thompson, 2004; Jensen, 1998), considera-se que os resultados obtidos para o Raven poderiam representar os reais efeitos do treinamento em MT e sua transferência para $\mathrm{G} f$. Esta questão será retomada na seção de Discussão.

Os efeitos analisados por variável moderadora são mostrados na Tabela 1, sempre que o tamanho da amostra permitiu (pelo menos 3 estudos por subgrupo). Uma análise dos resultados no Raven por variável moderadora indica efeitos significativos e positivos (ou seja, a favor do grupo que sofreu intervenção), mas a magnitude dos mesmos foi pequena. Os aumentos em $G f$ foram significativos para crianças/adolescentes e adultos, programas de treinamento de maior duração e treinamento em MT viso-espacial. Os demais resultados não alcançaram significância estatística. Análises de comparação entre os sub- 
A

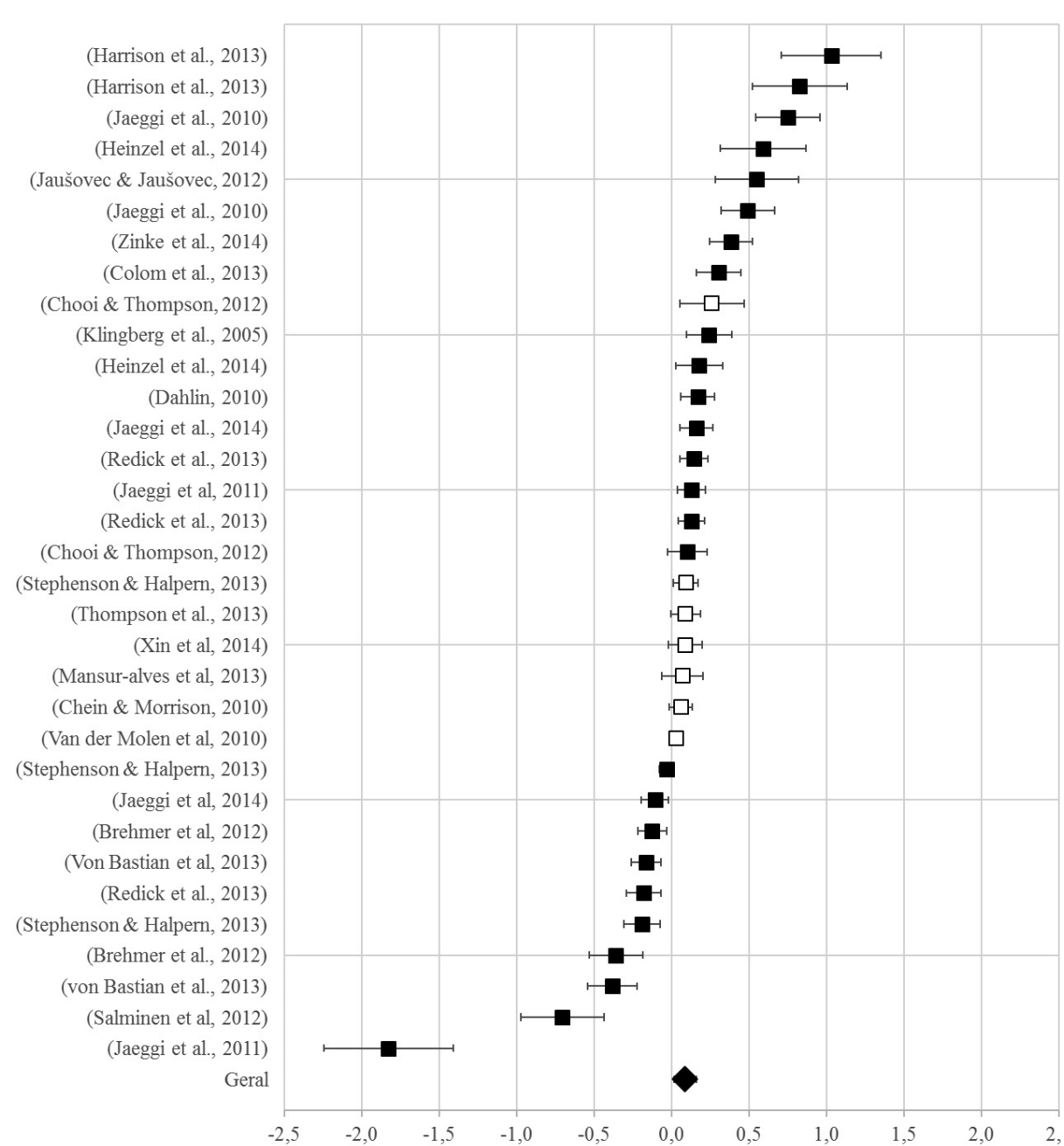

B
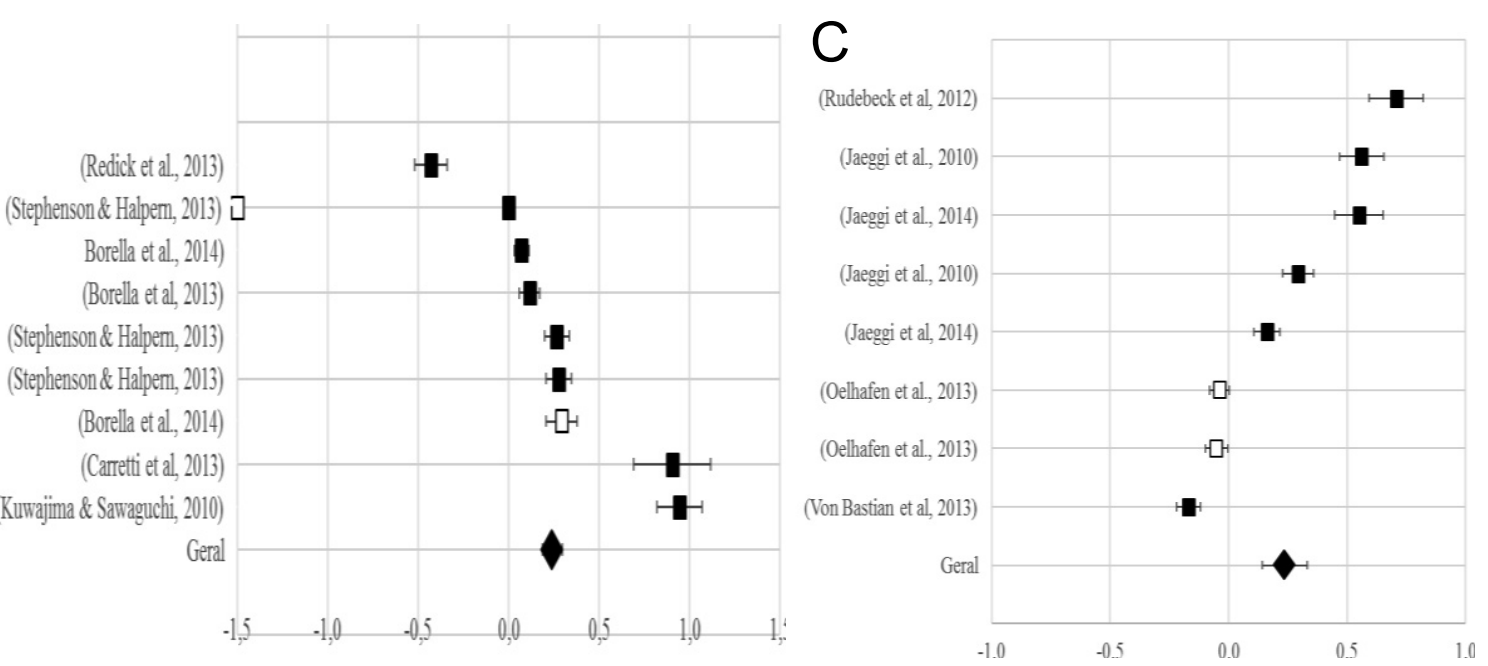

Figura 2. Gráfico de Floresta para efeitos do treinamento em MT no Raven (A) CFIT (B) e BOMAT (C), mostrando efeitos específicos de cada estudo. Os intervalos de confiança são representados pelas linhas horizontais. Marcadores não preenchidos indicam efeito não significativo.

grupos (teste $Z$ ) apontaram diferenças significativas apenas para crianças/adolescentes e adultos $(Z=130,37 ; p<0,001)$, em favor das primeiras.
Investigou-se, ainda, efeitos de longo prazo das intervenções realizadas. Foram encontrados dados de follow-up em 8 dos estudos que utiliza- 
Tabela 1

Análise da Homogeneidade dos Estudos Considerando as Variáveis Moderadoras para o Raven

\begin{tabular}{|c|c|c|c|c|}
\hline Variáveis Moderadoras & $N$ & G & Q & $\mathrm{I}^{2}$ \\
\hline \multicolumn{5}{|l|}{ Faixa etária } \\
\hline Crianças/adolescentes & 05 & $0,12^{* *}$ & \multirow{3}{*}{$164,16^{* *}$} & $-23,99$ \\
\hline Adultos & 22 & $0,15^{* *}$ & & 64,14 \\
\hline Idosos & 06 & $-0,27$ & & 65,66 \\
\hline \multicolumn{5}{|l|}{ Intensidade do treinamento } \\
\hline Até 8 horas & 10 & $-0,03$ & \multirow{2}{*}{$130,85^{* *}$} & 38,11 \\
\hline Acima de 8 horas & 23 & $0,12^{*}$ & & $-74,58$ \\
\hline \multicolumn{5}{|l|}{ Tipo de GC } \\
\hline Passivo & 09 & 0,26 & \multirow{2}{*}{$189,97^{* *}$} & 14,54 \\
\hline Ativo & 24 & 0,03 & & 77,23 \\
\hline \multicolumn{5}{|l|}{ Tipo de treinamento } \\
\hline MT viso-espacial & 05 & $0,12^{*}$ & \multirow{3}{*}{$15,59^{* *}$} & 61,87 \\
\hline MT Verbal & 06 & 0,13 & & 36,40 \\
\hline MT mista & 22 & 0,06 & & $-124,34$ \\
\hline \multicolumn{5}{|l|}{ Incentivo Material } \\
\hline Presente & 23 & 0,07 & \multirow{2}{*}{3,76} & $-40,92$ \\
\hline Ausente & 04 & 0,12 & & $-3,52$ \\
\hline
\end{tabular}

Nota. GC, grupo controle; MT, memória de trabalho. ${ }^{*} p<0,05 ;{ }^{* *} p<0,01$.

ram o Raven e em 3 dos estudos que utilizaram o CFIT. O tempo de intervalo do pós-teste imediato para ofollow-up variou entre 90 e 270 dias para o Raven (média $=170$ dias; $D P=96,12$ ). Para os três estudos que utilizaram o CFIT, o intervalo do pós-teste para o follow-up foi de 240 dias. O tamanho médio geral do efeito de longo prazo para o Raven foi de $-0,03$ (- 0,14 - 0,08; para Intervalo de Confiança de $95 \%$, com $p<0,05)$. A amostra foi composta por 261 indivíduos no grupo experimental (média $=32,62 ; D P=7,29)$ e 211 no grupo controle (média $=26,37 ; D P=7,40$ ). A heterogeneidade do grupo foi moderada, com $\mathrm{Q}=17,22(p<0,05)$ e I2=59,36\%. Já para o CFIT, o tamanho médio geral do efeito de longo prazo foi praticamente nulo $(0,0001)$, com heterogeneidade moderada entre os estudos, sendo $\mathrm{Q}=2,87(p<0,05)$ e I2 $=30,22 \%$ ( $\mathrm{n}$ do grupo experimental e do grupo controle $=58$, Média $=19,33$; $D P=1,15)$. É importante ressaltar que os três estudos com uso do CFIT foram publicados pelo mesmo grupo de pesquisadores principais (Borella et al., 2014; Borella et al., 2013), o que poderia indicar a presença dos mesmos tipos de viés metodológico. Assim, para que os resultados possam ser generalizados seria necessária a ampliação da amostra, com estudos realizados por grupos de pesquisa independentes que fizessem uso do CFIT.

\section{Discussão}

Pretendeu-se realizar uma análise crítica dos dados disponíveis na literatura visando lançar luz à controvérsia sobre a efetividade e transferência dos ganhos dos programas de treinamento em memória de trabalho para inteligência fluida. Ademais, objetivou-se identificar possíveis moderadores da transferência, de forma a compreender melhor quais as variantes metodológicas 
poderiam estar contribuindo mais efetivamente para os ganhos.

No que se refere à principal pergunta do presente estudo, referente aos ganhos dos programas de treinamento em MT para uma habilidade não diretamente treinada pelo programa (no presente caso, aquelas subjacentes ao construto de inteligência fluida), pode-se dizer que os resultados encontrados apontam que não há evidências sólidas suficientes de que o treinamento em MT produz ganhos imediatos e generalizados nas medidas de inteligência fluida aqui consideradas. Os tamanhos de efeito médios, embora tenham apontado para efeitos a favor do grupo experimental, foram pequenos $(0,09$ para o Raven e 0,24 para o CFIT e BOMAT). É importante considerar que não foi possível identificar viés de publicação para os estudos que utilizaram o CFIT e o BOMAT como medidas de $\mathrm{G} f$, já que o número de estudos para estas duas medidas foi menor do que 10. Dessa forma, não é possível saber se o tamanho de efeito médio encontrado se manteria na ausência de viés, haja vista que, caso o número de estudos com uso destas escalas fosse maior, aumentaria, também, o número de estudos com efeitos nulos ou negativos e, como consequência, o tamanho médio do efeito poderia ser menor que o encontrado no presente estudo. Dessa forma, considerando-se que: (a) os estudos com uso do Raven foram mais numerosos e não apresentaram viés de publicação; (b) o referido instrumento é um dos mais utilizados em estudos que se propõem a avaliar $G f$ e (c) o mesmo apresenta forte carga fatorial no fator geral de inteligência, é possível inferir que os resultados médios encontrados no Raven reflitam o tamanho de efeito mais provável em $G f$.

Os resultados encontrados nos estudos de follow-up indicam que os ganhos de longo prazo (no máximo 9 meses) tendem a ser nulos. Isso significa dizer que mesmo que haja um pequeno aumento das habilidades diretamente treinadas pelo programa ou efeitos indiretos destas em outras capacidades cognitivas associadas, estes efeitos não são mantidos ao longo do tempo. Uma das principais dificuldades dos programas de treinamento cognitivo, apontadas desde o seu advento nas décadas de 1960 e 1970 , reside no que é conhecido como fade-out effects (Hernstein \& Murray, 1994), ou seja, no esvanecimento dos benefícios obtidos com participação em programas de intervenção, tão logo estes programas se encerram. É possível argumentar que, com bases em princípios gerais de plasticidade cerebral, a indução da mesma requer suficiente repetição. Embora não haja um consenso que indique o significado de suficiente - em especial no que se refere a processos cognitivos complexos, como MT ou inteligência (Kleim \& Jones, 2008) - para que ocorra plasticidade em sistemas cerebrais com ganho em alguma função ou processo particular não somente a aquisição da mesma seria necessária, mas o uso contínuo daquele sistema ao longo do tempo. Seria este uso que faria com que o comportamento ou capacidade recém adquiridos não fossem perdidos na ausência do treinamento (Monfils, Plautz, \& Kleim, 2005). Se o indivíduo que passa por um programa de treinamento cognitivo não tem ou não cria oportunidades adequadas para uso do novo repertório cognitivo adquirido, o seu sistema voltaria ao estado inicial (antes do treino). Nesse sentido, é que tem sido defendida a importância de se considerar a validade ecológica das tarefas empregadas nos programas de treinamento e do ensino de estratégias cognitivas e metacognitivas de forma direta e consciente (Bahar-Fuchs, Clare, \& Woods, 2013; Green \& Bavelier, 2008: McDaniel \& Bugg, 2012). As estratégias treinadas e as tarefas utilizadas em laboratório possuem, em muitos casos, pouca semelhança com as demandas encontradas pelos sujeitos em seu cotidiano. Poder-se-ia ser mais relevante e eficiente treinar estratégias especificas (ensaio subvocal, organização, estabelecimento de relações, dentre outras) que pudessem ser aplicadas à solução de problemas diversos e que pudessem ser generalizadas para outras situações e atividades. McDaniel e Bugg (2012) enfatizam, ainda, que os programas de treinamento que possuem resultados positivos em nível da plasticidade dos sistemas cerebrais são aqueles cujas tarefas utilizadas para treinamento possuem repercussões diretas para a vida dos indivíduos treinados. Healy, Wohldmann, Parker e Bourne (2005) acreditam que uma solução seria optar pelo que eles 
chamam de prática distribuída nos programas de treinamento. A prática distribuída consiste em ministrar atividades que possam acelerar o desenvolvimento cognitivo ou promover mudanças intelectuais por um período mais extenso de tempo. Seria o mesmo que criar um programa de treinamento de MT, por exemplo, que pudesse ser aplicado durante todo o ano escolar, uma vez por semana, em casa e na escola. No caso específico do contexto brasileiro e da organização do nosso sistema de educação formal atual entende-se que seja praticamente impossível estabelecer um programa de treinamento baseado numa prática distribuída sem apoio do poder público ou de políticas mais efetivas de educação.

Partindo de uma análise otimista, poder-se-ia dizer que, embora pequeno, haveria sim um efeito de transferência do treino em MT para inteligência fluida que, em última instância e a longo prazo, poderia ter impacto positivo em indicadores sociais e educacionais (Buschkuehl \& Jaeggi, 2010; Chein \& Morrison, 2010). Contudo, este pequeno efeito poderia estar sujeito a várias outras interpretações, inclusive de falhas nos métodos de avaliação, não necessariamente indicando ganhos reais nesta capacidade. Esta hipótese é defendida por Hayes et al. (2015), que afirmam que os ganhos em escores de testes de inteligência podem ocorrer sem aumento em velocidade de processamento e capacidade, podendo indicar desenvolvimento de estratégias, inclusive de análise visual. Segundo eles, há duas maneiras de verificar se os ganhos em MT de fato se transferem para Gf. Uma delas é a inclusão de medidas múltiplas de $\mathrm{G} f$, o que permitiria extrair um fator latente. A segunda, que vem sendo utilizada por eles, é analisar o padrão de fixação ocular para inferir se o refinamento de estratégias poderia ser responsável pelos ganhos teste-reteste observados em $\mathrm{G} f$. Hayes et al. (2015) afirmam que este pequeno efeito observado em treinamentos de MT para $\mathrm{G} f$ poderiam ser explicados por desenvolvimento de estratégias de análise visual mais bem elaboradas. Seria, também, possível hipotetizar que o desenvolvimento de estratégias mais refinadas de análise visual poderia atuar como mediador dos ganhos observados. Apropriando-se da ideia de media- ção dos ganhos em programas de treinamento de MT em medidas de inteligência é importante destacar o papel dos recursos atencionais. A atenção pode ser entendida como um conjunto de processos que favorecem a seleção e distribuição dos recursos de processamento do sistema cognitivo, sendo, pois, em última instância importante para a eficiência do funcionamento cognitivo superior (Schweizer \& Moosbrugger, 2004). Diferentes tipos de atenção, mas especialmente a atenção executiva, têm sido associados tanto à memória de trabalho (distinção entre memória de curto-prazo e de trabalho) quanto à inteligência (Schweizer \& Koch, 2002; Schweizer, Moosbrugger, \& Goldhamme, 2005; Schweizer, Zimmermann, \& Koch, 2001). Ambos, atenção e MT, predizem desempenho em testes de inteligência, muito embora não se tenha clareza acerca da maneira como esta relação acontece, podendo haver contribuições não relacionadas ou interdependência. Schweizer e Moosbrugger (2004) testaram, através de análise fatorial confirmatória, diferentes modelos da relação entre MT, atenção e inteligência (medida pelas Matrizes Progressivas de Raven - Escala Avançada) em uma amostra de universitários e chegaram à conclusão de que a atenção contribui para o desempenho no teste de inteligência separadamente e através da memória de trabalho. Isto implica dizer que a habilidade de manter atenção em alto nível e coordenar diferentes recursos cognitivos é especialmente importante quando tarefas cognitivas complexas precisam ser finalizadas. Estendendo estes resultados para programas de treinamento é viável inferir que a atenção poderia ser um mediador dos ganhos cognitivos e que um treinamento atencional complementar (outros tipos de atenção) ao treino em memória de trabalho poderia potencializar os ganhos observados neste último. Green e Bavelier (2008) apontam para os resultados promissores que são encontrados em treinamentos formais e informais que utilizam de modelos multicomponentes de intervenção que inserem a atenção como componente essencial dos programas de intervenção. O presente estudo não nos permite afirmar nada sobre o papel da atenção como provável mediador de ganhos dos programas de 
treinamento de MT em inteligência, tampouco nos permite verificar a contribuição única e diferencial de programas de treinamento que foquem exclusivamente processos atencionais e seu impacto em medidas de inteligência fluida. Nesse sentido, até que os mecanismos envolvidos nesta transferência sejam mais bem descritos, não se pode afirmar com certeza que os efeitos dos treinos em MT se transferem para inteligência fluida, pelo menos, não diretamente, podendo ser inclusive, mediados por outras funções cognitivas ou estratégias utilizadas na resolução de problemas ou atenção.

A análise dos moderadores foi realizada apenas para o Raven. Um importante padrão emergiu: ganhos significativos para crianças-adolescentes em comparação com adultos, para treinamentos de maior duração e para tarefas de MT visuo-espacial. A plasticidade cerebral parece ser um importante fator para determinar ganhos em habilidades não-treinadas, especialmente para crianças e adolescentes. Plasticidade está relacionada a alterações neuroquímicas e estruturais no sistema nervoso que podem ser induzidas por treinamento ou experiências em ambientes cognitivamente enriquecidos. Embora a capacidade para estas alterações esteja presente em todas as fases da vida, na infância as respostas à estimulação ambiental são mais rápidas e parecem acontecer em maior magnitude do que em fases posteriores do desenvolvimento (Astle et al., 2015; Rosenzweig \& Bennett, 1996). Aliado às questões relativas à plasticidade, alguns autores têm sugerido que treinamentos de curta duração (mesmo que intensivos) não seriam suficientes para induzir plasticidade cerebral em uma habilidade tradicionalmente refratária à mudança, tal como a inteligência fluida (Astle et al., 2015; Chein \& Morrison, 2010; Melby-Lervåg \& Hulme, 2013, 2015). Isto parece corroborar os resultados aqui encontrados de que treinamentos de maior duração seriam mais eficazes. Seria, pois, necessária uma prática mais intensa não só para possibilitar as mudanças pretendidas, mas também para mantê-las. Rosenzweig e Bennett (1996) ressaltam que, muito embora ambientes e treinamentos possam não reverter toda uma série de déficits presentes no indivíduo, a estimulação precisa ser constante e os programas deveriam ser mantidos por mais tempo. Isto possibilitaria o desafio constante do sistema cognitivo e consolidaria os ganhos obtidos por mais tempo (von Bastian \& Eschen, 2016). Embora não seja possível saber, até o momento, qual seria a intensidade ideal dos treinamentos, programas de intervenção de curta duração podem não levar a ganhos em habilidades não-treinadas. Ademais, os resultados encontrados parecem indicar que os ganhos seriam de modalidade específica. Apenas as tarefas de memória de trabalho visuo-espacial produziram efeitos (mesmo que pequenos) no Raven. Embora o teste Raven não demande em todos os seus itens habilidades de processamento visuo-espaciais, alguns itens de algumas das séries do teste de matrizes saturam em componentes visuo-espaciais, os quais estão também presentes nas tarefas de MT visuo-espaciais incluídas nos programas de treinamento aqui analisados (Gignac, 2015; Hayes et al., 2015). Não obstante, para verificar se de fato os ganhos são de modalidade específica ou se seriam de domínio geral (no executivo central, por exemplo) seria importante mais uma vez incluir, como medida de eficácia, uma bateria de testes de inteligência fluida. Esta bateria deveria contemplar, senão todas, grande parte das operações características do construto de inteligência fluida, tais como reconhecimento e formação de conceitos, resolução de problemas, inferência e transformação de informações nas modalidades verbais e não-verbais, e não apenas um instrumento, como é usualmente feito.

Não obstante os resultados encontrados e discussões propostas, sabe-se que o presente estudo de revisão apresenta algumas limitações. Não foram realizadas buscas em bancos de dissertação e em anais de congressos, nem foi realizado contato com pesquisadores a fim de se obter o texto completo, quando este não estava disponível nas bases de dados acessadas. Assim sendo, os dados potencialmente ausentes poderiam apontar um viés em nossa análise das publicações. Ademais, devido ao pequeno número de estudos disponíveis na literatura e retidos para análise, não foi possível investigar possíveis efeitos diferenciais da transferência 
para $\mathrm{G} f$ do treino em MT para grupos clínicos distintos e também para crianças e adolescentes em diferentes idades e, portanto, em diferentes etapas do desenvolvimento cognitivo e neuronal. Neste último caso, não foi possível testar a hipótese de que os efeitos observados deveriam ser maiores para crianças em etapas tenras do desenvolvimento. As limitações apresentadas não comprometem o valor dos resultados encontrados, mas apontam para necessidade de revisões futuras que considerem e superem as limitações aqui listadas.

\section{Conclusões}

De forma geral, o presente estudo de revisão não encontrou evidências robustas de transferência dos ganhos para inteligência fluida dos treinamentos em memória de trabalho. Os resultados encontrados apontam para ganhos irrisórios e limitados a crianças com desenvolvimento típico. Ademais, a qualidade metodológica dos estudos incluídos e realizados acerca do tema é duvidosa. Não foi possível analisar diferenças no efeito médio entre amostras clínicas e não-clínicas e para diferentes tipos de prática de treinamento (distribuída e concentrada). Apenas três estudos incluíram grupos clínicos e somente quatro trabalharam com esquema de prática distribuída (intensa e contínua). Frequentemente se afirma que programas de treinamento em MT poderiam trazer benefícios para populações clínicas (Cortese et al., 2015). Não é possível saber se estudos futuros vão encontrar efeitos consistentes de transferência para medidas de inteligência fluida, mas até que a transferência dos ganhos para medidas de desempenho cognitivo e escolar (habilidades verbais e aritméticas) seja estudada de forma consistente, o uso clínico e disseminado de tais programas deve ser reduzido e realizado de forma mais cautelosa (Redick et al., 2015).

\section{Referências}

Ackerman, P. L., Beier, M. E., \& Boyle, M. O. (2005). Working memory and intelligence: The same or different constructs? Psychological Bulletin, 131(1), 30. doi:10.1037/0033-2909.131.1.30
Alloway, T. P., Bibile, V., \& Lau, G. (2013). Computerized working memory training: Can it lead to gains in cognitive skills in students? Computers in Human Behavior, 29(3), 632-638. doi:10.1016/j.chb.2012.10.023

Astle, D. E., Barnes, J. J., Baker, K., Colclough, G. L., \& Woolrich, M. W. (2015). Cognitive training enhances intrinsic brain connectivity in childhood. The Journal of Neuroscience, 35(16), 6277-6283. doi:10.1523/ JNEUROSCI.4517-14.2015

Au, J., Sheehan, E., Tsai, N., Duncan, G.J., Buschkuehl, M., \& Jaeggi, S. M. (2015). Improving fluid intelligence with training on working memory: A meta-analysis. Psychonomic Bulletin \& Review, 22(2), 366-377. doi:10.3758/s13423014-0699-x

Baddeley, A. (2003). Working memory: Looking back and looking forward. Nature Reviews Neuroscience, 4(10), 829-839. doi:10.1038/ nrn1201

Bahar-Fuchs, A., Clare, L., \& Woods, B. (2013). Cognitive training and cognitive rehabilitation for persons with mild to moderate dementia of the Alzheimer's or vascular type: A review. Alzheimer's Research \& Therapy, 5(4), 1-14. Retrieved from http://alzres.com/content/5/4/35

Barnet, S. M., \& Ceci, S. J. (2002). When and where do we apply what we learn?: A taxonomy for far transfer. Psychological Bulletin, 128(4), 612. doi:10.1037/0033-2909.128.4.612

*Borella, E., Carretti, B., Cantarella, A., Riboldi, F., Zavagnin, M., \& De Beni, R. (2014). Benefits of training visuospatial working memory in youngold and old-old. Developmental Psychology, 50(3), 714-727. doi:10.1037/a0034293

*Borella, E., Carretti, B., Riboldi, F., \& De Beni, R. (2010). Working memory training in older adults: Evidence of transfer and maintenance effects. Psychology and Aging, 25(4), 767-778. doi:10.1037/a0020683

*Borella, E., Carretti, B., Zanoni, G., Zavagnin, M., \& De Beni, R. (2013). Working memory training in old age: An examination of transfer and maintenance effects. Archives of Clinical Neuropsychology: The Official Journal of the National Academy of Neuropsychologists, 28(4), 331-347. doi:10.1093/arclin/act020

Borenstein, M., Hedges, L. V, Higgins, J. P. T., \& Rothstein, H. R. (2009). Subgroup analyses. In M. Borenstein, L. V Hedges, J. P. T. Higgins, 
\& H. R. Rothstein (Eds.), Introduction to MetaAnalysis. Chichester, UK: John Wiley \& Sons.

*Brehmer, Y., Westerberg, H., \& Bäckman, L. (2012, March). Working-memory training in younger and older adults: Training gains, transfer, and maintenance. Frontiers in Human Neuroscience, 6, 63. doi:10.3389/fnhum.2012.00063

Buschkuehl, M., \& Jaeggi, S. M. (2010). Improving intelligence: A literature review. Swiss Medical Weekly, 140(19-20), 266-272. doi:10.1.1.613.2047

Burt, C. (1962). Francis Galton and his contributions to psychology. British Journal of Statistical Psychology, 15(1), 1-49. doi:10.1111/j.2044-8317.1962.tb00081.x

Carpenter, P. A., Just, M. A., \& Shell, P. (1990). What one intelligence test measures: A theoretical account of the processing in the Raven Progressive Matrices Test. Psychological review, 97(3), 404. doi:10.1037/0033-295X.97.3.404

*Carretti, B., Borella, E., Fostinelli, S., \& Zavagnin, M. (2013). Benefits of training working memory in amnestic mild cognitive impairment: Specific and transfer effects. International Psychogeriatrics / IPA, 25(4), 617-626. doi:10.1017/ S1041610212002177

*Chein, J. M., \& Morrison, A. B. (2010). Expanding the mind's workspace: Training and transfer effects with a complex working memory span task. Psychonomic Bulletin \& Review, 17(2), 193-199. doi:10.3758/PBR.17.2.193

*Chooi, W.-T., \& Thompson, L. A. (2012). Working memory training does not improve intelligence in healthy young adults. Intelligence, 40(6), 531-542. doi:10.1016/j.intell.2012.07.004

Colom, R., Jung, R. E., \& Haier, R. J. (2007). General intelligence and memory span: Evidence for a common neuroanatomic framework. Cognitive Neuropsychology, 24(8), 867-878. doi:10.1080/02643290701781557

*Colom, R., Román, F. J., Abad, F. J., Shih, P. C., Privado, J., Froufe, M., ...Jaeggi, S. M. (2013). Adaptive n-back training does not improve fluid intelligence at the construct level: Gains on individual tests suggest that training may enhance visuospatial processing. Intelligence, 41(5), 712-727. doi:10.1016/j.intell.2013.09.002

Cortese, S., Ferrin, M., Brandeis, D., Buitelaar, J., Daley, D., Dittmann, R. W., ...Zuddas, A. (2015). Cognitive training for attention- deficit/hyperactivity disorder: Meta-analysis of clinical and neuropsychological outcomes from randomized controlled trials. Journal of the American Academy of Child \& Adolescent Psychiatry, 54(3), 164-174. doi:10.1016/j. jaac.2014.12.010

*Dahlin, K. I. E. (2010). Effects of working memory training on reading in children with special needs. Reading and Writing, 24(4), 479-491. doi:10.1007/s11145-010-9238-y

Deary, I. J., Penke, L., \& Johnson, W. (2010). The neuroscience of human intelligence differences. Nature Reviews Neuroscience, 11(3), 201-211. doi:10.1038/nrn2793

Flynn, J. R. (1987). Massive IQ gains in 14 nations: What IQ tests really measure. Psychological Bulletin, 101(2), 171-191. doi:10.1037/00332909.101.2.171

Flynn, J. R. (2006). Thethering the elephant: Capital cases, IQ, and the Flynn effect. Psychology, Public Policy, and Law, 12(2), 170-189. doi:10.1037/1076-8971.12.2.170

Gignac, G. E. (2015). Raven's is not a pure measure of general intelligence: Implications for $\mathrm{g}$ factor theory and the brief measurement of g. Intelligence, 52, 71-79. doi:10.1016/j.intell.2015.07.006

Gray, J. R., \& Thompson, P. M. (2004). Neurobiology of intelligence: Science and ethics. Nature Reviews Neuroscience, 5(6), 471-482. doi:10.1038/nrn1405

Green, C. S., \& Bavelier, D. (2008). Exercising your brain: A review of human brain plasticity and training-induced learning. Psychology and Aging, 23(4), 692-701. doi:10.1037/a0014345

*Harrison, T. L., Shipstead, Z., Hicks, K. L., Hambrick, D. Z., Redick, T. S., \& Engle, R. W. (2013). Working memory training may increase working memory capacity but not fluid intelligence. Psychological Science, 24(12), 24092019. doi:10.1177/0956797613492984

Hayes, T. R., Petrov, A. A., \& Sederberg, P. B. (2015). Do we really become smarter when our fluid-intelligence test scores improve?. Intelligence, 48, 1-14. doi:10.1016/j.intell.2014.10.005

Healy, A. F., Wohldmann, E. L., Parker, J. T., \& Bourne, L. E. (2005). Skill training, retention, and transfer: The effects of a concurrent secondary task. Memory \& Cognition, 33, 1457-1471. doi:10.3758/BF03193378 
Hedges, L., \& Olkin, I. (1985). Statistical models for meta-analysis. New York: Academic

*Heinzel, S., Schulte, S., Onken, J., Duong, Q.-L., Riemer, T. G., Heinz, A., ...Rapp, M. A. (2014). Working memory training improvements and gains in non-trained cognitive tasks in young and older adults. Neuropsychology, Development, and Cognition. Section B, Aging, Neuropsychology and Cognition, 21(2), 146-173. doi: 10.1080/13825585.2013.790338

Higgins, J. P. T., Thompson, S. G., Deeks, J. J., \& Altman, D. G. (2003). Measuring inconsistency in meta-analyses. BMJ: British Medical Journal, 327(7414), 557-560. doi:10.1136/ bmj.327.7414.557

Ilkowska, M., \& Engle, R. W. (Eds.). (2010) Working memory capacity and self-regulation, in handbook of personality and selfregulation. Oxford, UK: Wiley-Blackwell. doi:10.1002/9781444318111.ch12

Jaeggi, S. M., Buschkuehl, M., Jonides, J., \& Perrig, W. J. (2008). Improving fluid intelligence with training on working memory. Proceedings of the National Academy of Sciences, 105(19), 68296833. doi:10.1073/pnas.0801268105

*Jaeggi, S. M., Buschkuehl, M., Jonides, J., \& Shah, P. (2011). Short- and long-term benefits of cognitive training. Proceedings of the National Academy of Sciences of the United States of America, 108(25), 10081-10086. doi:10.1073/ pnas. 1103228108

*Jaeggi, S. M., Buschkuehl, M., Shah, P., \& Jonides, J. (2014). The role of individual differences in cognitive training and transfer. Memory \& Cognition, 42(3), 464-480. doi:10.3758/s13421013-0364-z

*Jaeggi, S. M., Studer-Luethi, B., Buschkuehl, M., Su, Y.-F., Jonides, J., \& Perrig, W. J. (2010). The relationship between n-back performance and matrix reasoning - Implications for training and transfer. Intelligence, 38(6), 625-635. doi:10.1016/j.intell.2010.09.001

*Jaušovec, N., \& Jaušovec, K. (2012). Working memory training: Improving intelligence--Changing brain activity. Brain and Cognition, 79(2), 96106. doi:10.1016/j.bandc.2012.02.007

Jensen, A.R. (1981). Raising the IQ: The Ramey and Haskins Study. Intelligence, 5, 29-40.

Jensen, A.R. (1998). The g factor. London: Praeger.
Jung, R. E., \& Haier, R. J. (2007). The Parieto-Frontal Integration Theory (P-FIT) of intelligence: Converging neuroimaging evidence. Behavioral and Brain Sciences, 30(02), 135-154. doi:10.1017/ S0140525X07001185

Kane, M. J., \& Engle, R. W. (2002). The role of prefrontal cortex in working-memory capacity, executive attention, and general fluid intelligence: An individual-differences perspective. Psychonomic Bulletin \& Review, 9(4), 637-671. doi:10.3758/BF03196323

Kleim, J. A., \& Jones, T. A. (2008). Principles of experience-dependent neural plasticity: implications for rehabilitation after brain damage. Journal of Speech, Language, and Hearing Research, 51, 225-239. doi:1092-4388/08/5101-S225

Klingberg, T. (2010). Training and plasticity of working memory. Trends in Cognitive Sciences, 14(7), 317-324. doi:10.1016/j.tics.2010.05.002

*Klingberg, T., Fernell, E., Olesen, P. J., Johnson, M., Gustafsson, P. E. R., Om, K. D., ... Westerberg, H. (2005). Computerized Training of Working Memory in Children with ADHD - A randomized controlled trial. Journal of the American Academy of Child and Adolescent Psychiatry, 44(2), 177-186. doi:10.1097/00004583-200502000-00010

*Klingberg, T., Forssberg, H., \& Westerberg, H. (2002). Training of working memory in children with ADHD. Journal of Clinical and Experimental Neuropsychology, 24(6), 781-791. doi:10.1076/jcen.24.6.781.8395

*Kuwajima, M., \& Sawaguchi, T. (2010). Similar prefrontal cortical activities between general fluid intelligence and visuospatial working memory tasks in preschool children as revealed by optical topography. Experimental Brain Research, 206(4), 381-397. doi:10.1007/ s00221-010-2415-z

Kyllonen, P. C., \& Christal, R. E. (1990). Reasoning ability is (little more than) working-memory capacity?!. Intelligence, 14(4), 389-433. doi:10.1016/S0160-2896(05)80012-1

Li, Y., Liu, Y., Li, J., Qin, W., Li, K., Yu, C., \& Jiang, T. (2009). Brain anatomical network and intelligence. PLoS Comput Biol, 5(5), e1000395. doi:10.1371/journal.pcbi.1000395

Li, T., \& Shotton, K. (2013). Conventional occlusion versus pharmacologic penalization for amblyo- 
pia. NIH Public Access, (4). doi:1002/14651858. CD006460.pub2.Conventional

Hernstein, R. J., \& Murray, C. (1994). The Bell Curve: Intelligence and Class Structure in American Life. New York: Free Press.

*Mansur-Alves, M., Flores-Mendoza, C., \& Tierra-Criollo, C. J. (2013). Evidências preliminares da efetividade do treinamento cognitivo para melhorar a inteligência de crianças escolares. Psicologia: Reflexão e Crítica, (1981), 423-434. doi:10.1590/S0102-79722013000300001

McDaniel, M. A., \& Bugg, J. M. (2012). Memory training interventions: What has been forgotten? Journal of Applied Research in Memory and Cognition, 1, 45-50. Retrieved from http:// dx.doi.org/10.1016/j.jarmac.2011.11.002

McGrew, K. S. (2009). CHC theory and the human cognitive abilities project: Standing on the shoulders of the giants of psychometric intelligence research. Intelligence, 37(1), 1-10. doi:10.1016/j.intell.2008.08.004

Melby-Lervåg, M., \& Hulme, C. (2013). Is working memory training effective? A meta-analytic review. Developmental Psychology, 49(2), 270. doi:10.1037/a0028228

Melby-Lervåg, M., \& Hulme, C. (2015). There is no convincing evidence that working memory training is effective: A reply to Au et al. (2014) and Karbach and Verhaeghen (2014). Psychonomic Bulletin and Review, 23(1), 324-330. doi:10.3758/s13423-015-0862-z

Miller, E. K., \& Cohen, J. D. (2001). An integrative theory of pre-frontal cortex function. Annual Review of Neuroscience, 24, 167-202. doi:10.1146/ annurev.neuro.24.1.167

Monfils, M. H., Plautz, E. J., \& Kleim, J. A. (2005). In search of the motor engram: Motor map plasticity as a mechanism for encoding motor experience. Neuroscientist, 11, 471-483. doi:10.1177/1073858405278015

Neisser, U., Boodoo, G., Bouchard, T. J., Jr., Boykin, A., Brody, N., Ceci, S. J., ...Urbina, S. (1996). Intelligence: Knowns and unknowns. American Psychologist, 51(2), 77-101. doi:10.1037/0003066X.51.2.77

Neyeloff, J. L., Fuchs, S. C., \& Moreira, L. B. (2012). Meta-analyses and Forest plots using a Microsoft excel spreadsheet: Step-by-step guide focusing on descriptive data analysis. BMC Research Notes, 5(Mix), 52. doi:10.1186/1756-0500-5-52
Nisbett, R. E., Aronson, J., Blair, C., Dickens, W., Flynn, J., Halpern, D. F., \& Turkheimer, E. (2012). Intelligence: New findings and theoretical developments. American Psychologist, 67(2), 130-159. doi:10.1037/a0026699

Oberauer, K., Suß, H.-M., Schulze, R., Wilhelm, O., \& Wittmann, W. W. (2000). Working memory capacity facets of a cognitive ability construct. Personality and Individual Differences, 29, 1017-1045. doi:10.1016/S01918869(99)00251-2

*Oelhafen, S., Nikolaidis, A., Padovani, T., Blaser, D., Koenig, T., \& Perrig, W. J. (2013). Increased parietal activity after training of interference control. Neuropsychologia, 51(13), 2781-2790. doi:10.1016/j.neuropsychologia.2013.08.012

Orzechowski, J. (2010). Working memory capacity and individual differences in higher-level cognition. In A. Gruszka., G. Matthews., \& B. Szymura (Eds.), Handbook of Individual differences in cognition: Attention, memory, and executive control (pp. 353-368). London: Springer.

Pereira, M. G., \& Galvão, T. F. (2014). Heterogeneidade e viés de publicação em revisões sistemáticas. Epidemiologia e Serviços de Saúde, 23(4), 775778. doi:10.5123/S1679-49742014000400021

*Pugin, F., Metz, A. J., Stauffer, M., Wolf, M., Jenni, O. G., \& Huber, R. (2015). Working memory training shows immediate and longterm effects on cognitive performance in children and adolescents. F1000Research, 1-10. doi:10.12688/f1000research.3929

*Redick, T. S., Shipstead, Z., Harrison, T. L., Hicks, K. L., Fried, D. E., Hambrick, D. Z., ...Engle, R. W. (2013). No evidence of intelligence improvement after working memory training: A randomized, placebo-controlled study. Journal of Experimental Psychology. General, 142(2), 359-379. doi:10.1037/a0029082

Redick, T. S., Shipstead, Z., Wiemers, E. A., MelbyLervåg, M., \& Hulme, C. (2015). What's working in working memory training? An educational perspective. Educational Psychology Review, 27(4), 617-633. doi:10.1007/s10648015-9314-6

*Richmond, L. L., Morrison, A. B., Chein, J. M., \& Olson, I. R. (2011). Working memory training and transfer in older adults. Psychology and Aging, 26(4), 813-822. doi:10.1037/a0023631 
Rosenzweig, M. R., \& Bennett, E. L. (1996). Psychobiology of plasticity: Effects of training and experience on brain and behavior. Behavioural Brain Research, 78(1), 57-65. doi:10.1016/01664328(95)00216-2

*Rudebeck, S. R., Bor, D., Ormond, A., O’Reilly, J. X., \& Lee, A. C. H. (2012). A potential spatial working memory training task to improve both episodic memory and fluid intelligence. PloS One, 7(11), e50431. doi:10.1371/journal. pone. 0050431

Rushton, J. P. (1990). Sir Francis Galton, epigenetic rules, genetic similarity theory, and human life-history analysis. Journal of Personality, 58(1), 117-140. doi:10.1111/j.1467-6494.1990. tb00910.x

*Salminen, T., Strobach, T., \& Schubert, T. (2012, June). On the impacts of working memory training on executive functioning. Frontiers in Human Neuroscience, 6, 166. doi:10.3389/ fnhum.2012.00166

Schmiedek, F., Lövdén, M., \& Lindenberger, U. (2010). Hundred days of cognitive training enhance broad cognitive abilities in adulthood: Findings from the COGITO study. Frontiers in Aging Neuroscience, 2, 27. doi:10.3389/ fnagi.2010.00027

Schweizer, K. \& Koch, W. (2002). A revision of Cattell's Investment Theory Cognitive properties influencing learning. Learning and Individual Difference, 13, 57-82. doi:10.1016/S10416080(02)00062-6

Schweizer, K., \& Moosbrugger, H. (2004). Attention and working memory as predictors of intelligence. Intelligence, 32(4), 329-347. doi:10.1016/j.intell.2004.06.006

Schweizer, K., Moosbrugger, H., \& Goldhammer, F. (2005). The structure of the relationship between attention and intelligence. Intelligence, 33(6), 589-611. doi:10.1016/j.intell.2005.07.001

Schweizer, K., Zimmermann, P., \& Koch, W. (2001). Sustained attention, intelligence and the crucial role of perceptual processes. Learning and Individual Differences, 12, 271-286. doi:10.1016/ S1041-6080(01)00040-1

Shipstead, Z., Redick, T. S., \& Engle, R. W. (2012). Is working memory training effective?. Psychological Bulletin, 138(4), 628. doi:10.1037/ a0027473
Sprenger, A. M., Atkins, S. M., Bolger, D. J., Harbison, J. I., Novick, J. M., Chrabaszcz, J. S., ...Bunting, M. F. (2013). Training working memory: Limits of transfer. Intelligence, 41(5), 638-663. doi:10.1016/j.intell.2013.07.013

Stauffer, J. M., Ree, M. J., \& Caretta, T. R. (1996). Cognitive-components tests are not much more than g: An extension of Kyllonen's analysis. Journal of General Psychology, 123, 193-205. doi:10.1080/00221309.1996.9921272

*Stephenson, C. L., \& Halpern, D. F. (2013). Improved matrix reasoning is limited to training on tasks with a visuospatial component. Intelligence, 41(5), 341-357. doi:10.1016/j.intell.2013.05.006

Strenze, T. (2007). Intelligence and socioeconomic success: A meta-analytic review of longitudinal research. Intelligence, 35(5), 401-426. doi:10.1016/j.intell.2006.09.004

*Thompson, T. W., Waskom, M. L., Garel, K.-L. A., Cardenas-Iniguez, C., Reynolds, G. O., Winter, R., ...Gabrieli, J. D. E. (2013). Failure of working memory training to enhance cognition or intelligence. PloS One, 8(5), e63614. doi:10.1371/journal.pone.0063614

*Van der Molen, M. J., Van Luit, J. E. H., Van der Molen, M. W., Klugkist, I., \& Jongmans, M. J. (2010). Effectiveness of a computerised working memory training in adolescents with mild to borderline intellectual disabilities. Journal of Intellectual Disability Research : JIDR, 54(5), 433447. doi:10.1111/j.1365-2788.2010.01285.x

Von Bastian, C. C., \& Eschen, A. (2016). Does working memory training have to be adaptive?. Psychological research, 80(2), 181-194. doi:10.1007/s00426-015-0655-Z

*Von Bastian, C. C., Langer, N., Jäncke, L., \& Oberauer, K. (2013). Effects of working memory training in young and old adults. Memory \& Cognition, 41(4), 611-624. doi:10.3758/s13421012-0280-7

Wang, Z., Zhou, R., \& Shah, P. (2014). Spaced cognitive training promotes training transfer. Frontiers in Human Neuroscience, 8, 1-8. doi:10.3389/fnhum.2014.00217

Woolgar, A., Parr, A., Cusack, R., Thompson, R., Nimmo-Smith, I., Torralva, T., ...Duncan, J. (2010). Fluid intelligence loss linked to restricted regions of damage within frontal and parietal 
cortex. Proceedings of the National Academy of Sciences, 107(33), 14899-14902. doi:10.1073/ pnas. 1007928107

*Xin, Z., Lai, Z.-R., Li, F., \& Maes, J. H. R. (2014). Near- and far-transfer effects of working memory updating training in elderly adults. Applied Cognitive Psychology, 28(3), 403-408. doi:10.1002/acp.3011

*Zinke, K., Zeintl, M., Rose, N. S., Putzmann, J., Pydde, A., \& Kliegel, M. (2014). Working memory training and transfer in older adults: Effects of age, baseline performance, and training gains. Developmental Psychology, 50(1), 304-315. doi:10.1037/a0032982

\section{Apêndice A}

Tabela 1A

Caracterização dos Estudos Encontrados

\begin{tabular}{|c|c|c|c|c|c|c|c|c|}
\hline \multirow{2}{*}{ Referência } & \multicolumn{2}{|c|}{ Média de Idade ( $D P$ ) } & \multirow[t]{2}{*}{$\begin{array}{c}n \\
\text { GE }\end{array}$} & \multirow[t]{2}{*}{$\stackrel{n}{\mathbf{G C}}$} & \multirow[t]{2}{*}{ Incentivo } & \multirow{2}{*}{$\begin{array}{c}\text { Treino } \\
\text { em } \\
\text { dias }\end{array}$} & \multirow[t]{2}{*}{$\begin{array}{l}\text { Tipo de } \\
\text { treinamento }\end{array}$} & \multirow[t]{2}{*}{$\begin{array}{c}\text { Medida } \\
\text { de } G \boldsymbol{f}\end{array}$} \\
\hline & GE & GC & & & & & & \\
\hline (Klingberg et al., 2002) & $11(2)$ & $11,4(3)$ & 7 & 7 & NI & 21 & Combinado & Raven \\
\hline (Klingberg et al., 2005) & $9,8(1,4)$ & $9,7(1,3)$ & 22 & 22 & NI & 35 & Combinado & Raven \\
\hline (Dahlin, 2010) & NI & NI & 42 & 15 & NI & 35 & Combinado & Raven \\
\hline $\begin{array}{l}\text { (Kuwajima \& Sawaguchi, } \\
\text { 2010) }\end{array}$ & NI & NI & 20 & 40 & NI & 56 & Viso-espacial & CFIT \\
\hline $\begin{array}{l}\text { (Van der Molen, Van Luit, } \\
\text { Van der Molen, Klugkist, \& } \\
\text { Jongmans, 2010) }\end{array}$ & $15,32(0,69)$ & NI & 41 & 54 & Presente & 35 & Viso-espacial & Raven \\
\hline (Chein \& Morrison, 2010) & $20,1(\mathrm{NI})$ & $20,6(\mathrm{NI})$ & 21 & 21 & Presente & 30 & Combinado & Raven \\
\hline (Jaeggi et al., 2010) & $19(1,5)$ & $19(1)$ & 21 & 43 & Presente & 30 & Viso-espacial & $\begin{array}{l}\text { BOMAT/ } \\
\text { Raven }\end{array}$ \\
\hline (Jaeggi et al., 2010) & $19,1(1,2)$ & $19(1)$ & 25 & 43 & Presente & 35 & Combinado & $\begin{array}{l}\text { BOMAT/ } \\
\text { Raven }\end{array}$ \\
\hline (Borella et al., 2010) & $69(3,18)$ & $69,15(2,99)$ & 20 & 20 & $\mathrm{NI}$ & 14 & $\begin{array}{l}\text { Alfabético- } \\
\text { verbal }\end{array}$ & CFIT \\
\hline (Jaeggi et al., 2011) & $9,12(1,52)$ & $8,83(1,44)$ & 32 & 30 & NI & 30 & Viso-espacial & Raven \\
\hline $\begin{array}{l}\text { (Richmond, Morrison, Chein, } \\
\text { \& Olson, 2011) }\end{array}$ & NI & NI & 21 & 19 & NI & 35 & Combinado & Raven \\
\hline $\begin{array}{l}\text { (Salminen, Strobach, \& } \\
\text { Schubert, 2012) }\end{array}$ & $24,4(\mathrm{NI})$ & $24,5(\mathrm{NI})$ & 20 & 18 & Presente & 21 & Combinado & Raven \\
\hline $\begin{array}{l}\text { (Brehmer, Westerberg, \& } \\
\text { Bäckman, 2012) }\end{array}$ & $26,2(2,8)$ & $25,7(3,5)$ & 29 & 26 & Presente & 35 & Combinado & Raven \\
\hline (Brehmer et al., 2012) & $63,9(3,4)$ & $63,6(3,1)$ & 26 & 19 & Presente & 42 & Combinado & Raven \\
\hline (Chooi \& Thompson, 2012) & $20(\mathrm{NI})$ & $\mathrm{NI}$ & 9 & 15 & Presente & 21 & Combinado & Raven \\
\hline (Chooi \& Thompson, 2012) & $20(\mathrm{NI})$ & NI & 13 & 11 & Presente & 35 & Combinado & Raven \\
\hline (Jaušovec \& Jaušovec, 2012) & $20(\mathrm{NI})$ & 20 & 14 & 15 & Presente & 84 & Combinado & Raven \\
\hline
\end{tabular}

Recebida: $25 / 07 / 2016$

$1^{a}$ revisão: $1 \% 11 / 2016$

Aceite final: 28/11/2016 


\begin{tabular}{|c|c|c|c|c|c|c|c|c|}
\hline \multirow{2}{*}{ Referência } & \multicolumn{2}{|c|}{ Média de Idade ( $D P$ ) } & \multirow[t]{2}{*}{$\begin{array}{c}n \\
\text { GE }\end{array}$} & \multirow[t]{2}{*}{$\begin{array}{c}n \\
\text { GC }\end{array}$} & \multirow{2}{*}{ Incentivo } & \multirow{2}{*}{$\begin{array}{c}\text { Treino } \\
\text { em } \\
\text { dias }\end{array}$} & \multirow[t]{2}{*}{$\begin{array}{l}\text { Tipo de } \\
\text { treinamento }\end{array}$} & \multirow[t]{2}{*}{$\begin{array}{c}\text { Medida } \\
\text { de } G f\end{array}$} \\
\hline & GE & GC & & & & & & \\
\hline (Oelhafen et al., 2013) & NI & NI & 14 & 8 & Presente & 21 & Combinado & BOMAT \\
\hline (Oelhafen et al., 2013) & NI & $\mathrm{NI}$ & 14 & 8 & Presente & 21 & Combinado & BOMAT \\
\hline (Von Bastian et al., 2013) & $23(4)$ & $23(4)$ & 34 & 32 & Presente & 30 & Combinado & $\begin{array}{l}\text { Raven/ } \\
\text { BOMAT, }\end{array}$ \\
\hline (Von Bastian et al., 2013) & $68(4)$ & $69(3)$ & 27 & 30 & Presente & 30 & Combinado & $\begin{array}{l}\text { Raven/ } \\
\text { BOMAT }\end{array}$ \\
\hline (Colom et al., 2013) & $18,04(0,9)$ & $18,2(1,2)$ & 28 & 28 & NI & 84 & Combinado & Raven \\
\hline (Harrison et al., 2013) & NI (NI) & $\mathrm{NI}$ & 21 & 17 & Presente & 30 & Combinado & Raven \\
\hline (Harrison et al., 2013) & NI & NI & 17 & 17 & Presente & 35 & Combinado & Raven \\
\hline (Redick et al., 2013) & $21,1(2,7)$ & $20,7(2,5)$ & 24 & 29 & NI & 30 & Combinado & $\begin{array}{l}\text { Raven/ } \\
\text { CFIT }\end{array}$ \\
\hline (Sprenger et al., 2013) & NI & NI & 34 & 37 & Presente & 42 & $\begin{array}{l}\text { Alfabético- } \\
\text { verbal }\end{array}$ & Raven \\
\hline (Sprenger et al., 2013) & NI & $\mathrm{NI}$ & 33 & 37 & Presente & 42 & Viso-espacial & Raven \\
\hline $\begin{array}{l}\text { (Stephenson \& Halpern, } \\
\text { 2013) }\end{array}$ & NI & NI & 28 & 28 & Presente & 30 & Combinado & $\begin{array}{l}\text { Raven/ } \\
\text { CFIT }\end{array}$ \\
\hline $\begin{array}{l}\text { (Stephenson \& Halpern, } \\
\text { 2013) }\end{array}$ & NI & NI & 29 & 28 & Presente & 35 & Viso-espacial & $\begin{array}{l}\text { Raven/ } \\
\text { CFIT }\end{array}$ \\
\hline $\begin{array}{l}\text { (Stephenson \& Halpern, } \\
\text { 2013) }\end{array}$ & NI & NI & 25 & 28 & Presente & 42 & $\begin{array}{l}\text { Alfabético- } \\
\text { verbal }\end{array}$ & $\begin{array}{l}\text { Raven/ } \\
\text { CFIT }\end{array}$ \\
\hline (Thompson et al., 2013) & $21,2(\mathrm{NI})$ & $21,3(\mathrm{NI})$ & 20 & 19 & Presente & 30 & Combinado & Raven \\
\hline $\begin{array}{l}\text { (Mansur-Alves, Flores- } \\
\text { Mendoza, \& Tierra-Criollo, } \\
\text { 2013) }\end{array}$ & NI & NI & 8 & 8 & ausente & 84 & $\begin{array}{l}\text { Alfabético- } \\
\text { verbal }\end{array}$ & Raven \\
\hline (Borella et al., 2013) & $79,22(3,49)$ & $79,17(2,95)$ & 18 & 18 & $\mathrm{NI}$ & 21 & $\begin{array}{l}\text { Alfabético- } \\
\text { verbal }\end{array}$ & CFIT \\
\hline $\begin{array}{l}\text { (Carretti, Borella, Fostinelli, } \\
\text { \& Zavagnin, 2013) }\end{array}$ & $71,8(2,2)$ & $70,6(2,63)$ & 10 & 10 & NI & 21 & $\begin{array}{l}\text { Alfabético- } \\
\text { verbal }\end{array}$ & CFIT \\
\hline (Heinzel et al., 2014) & $25,9(1,9)$ & $25,6(2,1)$ & 15 & 15 & Presente & 30 & $\begin{array}{l}\text { Alfabético- } \\
\text { verbal }\end{array}$ & Raven \\
\hline (Heinzel et al., 2014) & $66,07(4,7)$ & $65,6(3,9)$ & 15 & 15 & Presente & 35 & $\begin{array}{l}\text { Alfabético- } \\
\text { verbal }\end{array}$ & Raven \\
\hline (Borella et al., 2014) & $69,9(2,79)$ & $69,55(2,89)$ & 20 & 20 & NI & 14 & Viso-espacial & Cattell \\
\hline (Borella et al., 2014) & $79,6(2,28)$ & $79,7(2,3)$ & 20 & 20 & NI & 14 & Viso-espacial & Cattell \\
\hline (Xin, Lai, Li, \& Maes, 2014) & $70(5,87)$ & $69(7,01)$ & 15 & 14 & Presente & 21 & Combinado & Raven \\
\hline (Zinke et al., 2014) & $75,7(8,4)$ & $77,7(7,9)$ & 40 & 40 & ausente & 21 & Combinado & Raven \\
\hline $\begin{array}{l}\text { (Jaeggi, Buschkuehl, Shah, \& } \\
\text { Jonides, 2014) }\end{array}$ & NI & NI & 25 & 27 & ausente & 30 & Combinado & $\begin{array}{l}\text { Raven/ } \\
\text { BOMAT }\end{array}$ \\
\hline (Jaeggi et al., 2014) & NI & $\mathrm{NI}$ & 26 & 27 & ausente & 30 & $\begin{array}{l}\text { Alfabético- } \\
\text { verbal }\end{array}$ & $\begin{array}{l}\text { Raven/ } \\
\text { BOMAT }\end{array}$ \\
\hline
\end{tabular}

Nota. GC - Grupo Controle; GE - Grupo Experimental; NI - Não Informado. 


\section{Apêndice $B$}

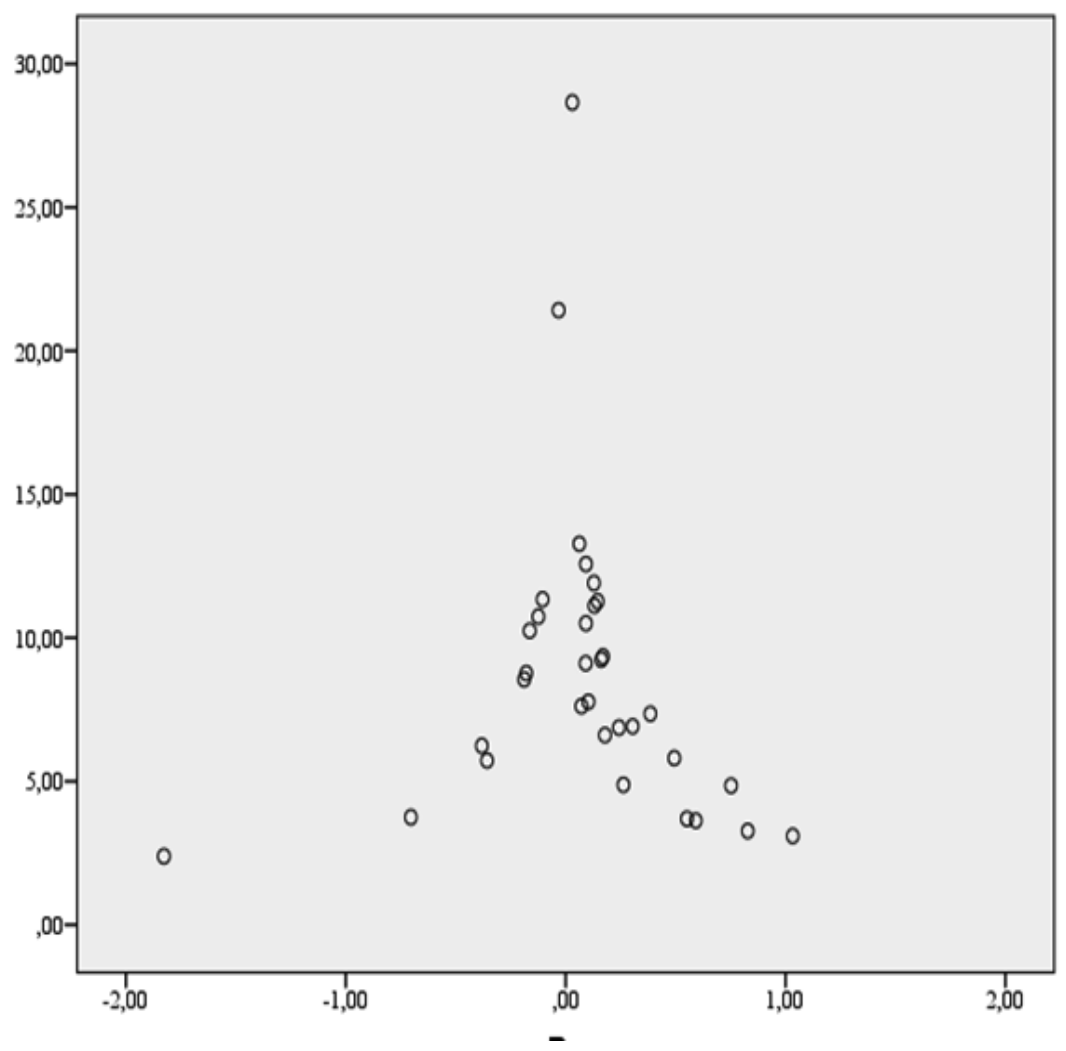

Figura 1B. Gráfico de Funil para o teste Raven. 